\title{
On the rationality of Kawamata log terminal singularities in positive characteristic
}

\author{
Christopher Hacon and Jakub Witaszek
}

\begin{abstract}
We show that there exists a natural number $p_{0}$ such that any three-dimensional Kawamata log terminal singularity defined over an algebraically closed field of characteristic $p>p_{0}$ is rational and in particular Cohen-Macaulay.
\end{abstract}

\section{Introduction}

One of the main goals of algebraic geometry is to understand the structure of smooth projective varieties. The techniques of the minimal model program, MMP for short, play a fundamental role in the pursuit of this objective. Unluckily, starting from dimension three, to apply the techniques of the MMP it is necessary to consider varieties with mild singularities. From the point of view of the MMP, the most natural class of singularities is that of Kawamata log terminal (klt) singularities, which includes canonical and terminal singularities. In characteristic zero, a large part of the MMP for varieties with klt singularities is known to hold (see [BCHM10]). In this context, it is of course essential to have a good understanding of the properties of klt singularities. Perhaps the most important of these properties is the fact that klt singularities are rational and, in particular, Cohen-Macaulay. This fundamental fact was first proved by Elkik for canonical singularities and was later generalized to klt singularities; see, for example, [KM98, Theorem 5.22] and references therein.

The main technical tool used in the proof of the results of the MMP (and in the proof of Elkik's result) is the famous Kawamata-Viehweg vanishing, which is known to fail in positive characteristic and dimension at least two. So it is not surprising that the situation in positive characteristic $p>0$ is much more complicated. In spite of this, it is known that the klt MMP is valid in dimension two [Tan14] and dimension three in characteristic $p>5$ [HX15, CTX15, Bir16, BW17]. It is natural to wonder if in positive characteristic, klt singularities are rational. In dimension two, this is known to hold for all characteristics; however, recently, it was shown that this does not hold when $p=2$ and the dimension is at least three [GNT15, Kov18, CT16] (see Remark 5.3). On the positive side, it is known that klt threefold singularities are Wittrational when $p>5$ [GNT15]. This interesting result allows for the extension of Esnault's results on counting points over finite fields to singular varieties. Note that rational singularities are

Received 25 August 2017, accepted in final form 24 August 2018.

2010 Mathematics Subject Classification 14E30, 14J17, 13A35.

Keywords: Kawamata log terminal singularities, rational singularities, positive characteristic.

This journal is (C) Foundation Compositio Mathematica 2019. This article is distributed with Open Access under the terms of the Creative Commons Attribution Non-Commercial License, which permits non-commercial reuse, distribution, and reproduction in any medium, provided that the original work is properly cited. For commercial re-use, please contact the Foundation Compositio Mathematica.

The first author was partially supported by NSF research grants nos DMS-1300750, DMS-1265285 and by a grant from the Simons Foundation, Award Number 25620. The second author was supported by the Engineering and Physical Sciences Research Council [EP/L015234/1]. 


\section{ON THE RATIONALITY OF KLT SINGULARITIES}

Witt-rational but the converse is not true. Furthermore, it is known that strongly F-regular singularities are rational. Note that F-regular singularities are klt, but klt threefold singularities need not be strongly F-regular even in large characteristic [CTW18].

The goal of this paper is to show the following result.

THEOREM 1.1. There exists a natural number $p_{0}>0$ such that for any three-dimensional Kawamata log terminal pair $(X, \Delta)$ defined over an algebraically closed field of characteristic $p>p_{0}$, we have that $X$ has rational and, in particular, Cohen-Macaulay singularities. Moreover, if $X$ is $\mathbb{Q}$-factorial, then, for any divisor $D$, the sheaf $\mathcal{O}_{X}(D)$ is Cohen-Macaulay.

It is not known whether the above theorem holds or is false if we just assume that $p>2$. The main tool in the proof of this result is the Kawamata-Viehweg vanishing for log del Pezzo surfaces in large characteristic. The constant $p_{0}$ comes from this result.

Theorem 1.2 ([CTW17, Theorem 1.2]). There exists a constant $p_{0}>0$ with the following property.

Let $X$ be a projective surface of Fano type defined over an algebraically closed field of characteristic $p>p_{0}$. Let $\Delta$ be an effective $\mathbb{Q}$-divisor such that $(X, \Delta)$ is klt, and let $L$ be a Weil divisor for which $L-\left(K_{X}+\Delta\right)$ is nef and big. Then $H^{i}\left(X, \mathcal{O}_{X}(L)\right)=0$ for $i>0$.

We say that a surface $X$ is of Fano type if there exists a $\mathbb{Q}$-divisor $B$ such that $(X, B)$ is klt and $-\left(K_{X}+B\right)$ is ample. Note that the Kawamata-Viehweg vanishing does not hold for rational surfaces in general even in large characteristic [CT18].

Let us also note that it has been recently shown in [Kov17] that Cohen-Macaulay klt singularities are rational. The results from that paper allowed us to simplify the proof of Theorem 1.1.

One possible application of these results stems from the minimal model program in mixed characteristic. Given a positive-characteristic variety $X$ which lifts to characteristic zero and a basic operation of the MMP $f: X \rightarrow Y$ such as a contraction or a flip, it is natural to ask if $f$ lifts to characteristic zero as well. As a corollary of Theorem 1.1, we show that this holds for divisorial and flipping contractions of threefolds in large characteristic (see Corollary 5.1). Unfortunately, we do not know how to deal with the case of flips.

The article is organised as follows. In Section 2, we discuss rational and Cohen-Macaulay singularities, strong F-regularity, purely log terminal (plt) blow-ups, and liftings to the rings of Witt vectors. In Section 3, we prove the existence of certain short exact sequences on plt threefolds (see Subsection 1.1). In Section 4, we finish the proof of Theorem 1.1. In Section 5, we explore applications to the MMP in mixed characteristic.

\subsection{Sketch of the proof}

Assume that $X$ is $\mathbb{Q}$-factorial. Consider a plt blow-up (Proposition 2.11) at a closed point $x \in X$, that is, a birational morphism $f: Y \rightarrow X$ with an irreducible exceptional divisor $S$ such that

- $(Y, S)$ is plt and $\mathbb{Q}$-factorial,

- $-\left(K_{Y}+S\right)$ is $f$-ample,

- $f$ is an isomorphism over $X \backslash\{x\}$.

By adjunction, there exists a $\mathbb{Q}$-divisor $\Delta_{\text {diff }}$ such that $\left(S, \Delta_{\text {diff }}\right)$ is a klt log del Pezzo pair.

When $S$ is Cartier, one can apply the following strategy (cf. [GNT15] and [CT16]). For every $k \geqslant 0$, consider

$$
0 \rightarrow \mathcal{O}_{Y}(-(k+1) S) \rightarrow \mathcal{O}_{Y}(-k S) \rightarrow \mathcal{O}_{S}\left(G_{k}\right) \rightarrow 0
$$




\section{HACON AND J. WitASZEK}

where $G_{k} \sim-\left.k S\right|_{S}$. Since

$$
G_{k} \sim_{\mathbb{Q}} K_{S}+\Delta_{\text {diff }} \underbrace{-\left(K_{S}+\Delta_{\text {diff }}\right)+G_{k}}_{\text {ample }},
$$

by Theorem 1.2, we have that $H^{1}\left(S, \mathcal{O}_{S}\left(G_{k}\right)\right)=0$ for every $k \geqslant 0$, and so $R^{1} f_{*} \mathcal{O}_{Y}(-(k+1) S) \rightarrow$ $R^{1} f_{*} \mathcal{O}_{Y}(-k S)$ is surjective. Since $-S$ is $f$-ample, $R^{1} f_{*} \mathcal{O}_{Y}(-k S)=0$ for $k \gg 0$ by the Serre vanishing theorem, which implies that $R^{1} f_{*} \mathcal{O}_{Y}=0$ as well. By [HX15], one sees that $Y$ has F-regular singularities along $S$ and hence $Y$ has rational singularities along $S$. Therefore, if $X$ is Cohen-Macaulay, then it has rational singularities at $x$. The fact that $X$ is Cohen-Macaulay at $x$ follows by a similar argument.

When $S$ is not Cartier, the above short exact sequence may fail to be exact; however, we show the existence of the following short exact sequence (Proposition 3.1):

$$
0 \rightarrow \mathcal{O}_{Y}(-(k+1) S) \rightarrow \mathcal{O}_{Y}(-k S) \rightarrow \mathcal{O}_{S}\left(G_{k}\right) \rightarrow 0
$$

where $G_{k}$ is a Weil divisor satisfying $G_{k} \sim_{\mathbb{Q}}-\left.k S\right|_{S}-\Delta_{k}$ for some effective $\mathbb{Q}$-divisor $\Delta_{k}$ on $S$ such that $\Delta_{k} \subseteq \Delta_{\text {diff }}$. Therefore, we can apply the same argument as above with minor changes.

When $X$ is not $\mathbb{Q}$-factorial, we apply Propositions 2.11 and 2.12 and the relative F-inversion of adjunction (Lemma 2.10).

\section{Preliminaries}

We say that a scheme $X$ is a variety if it is integral, separated, and of finite type over a field $k$. Throughout this paper, $k$ is an arbitrary field of positive characteristic $p>0$. For a $k$-variety $X$, we denote the relative canonical divisor $K_{X / \text { Spec } k}$ by $K_{X}$ (see [Kol13, Definition 1.6]).

We refer to [KM98] for basic definitions in birational geometry, and to [Kol13] whenever $k$ is not algebraically closed. We say that $(X, \Delta)$ is a $\log$ pair if $X$ is normal, $\Delta$ is an effective $\mathbb{Q}$-divisor, and $K_{X}+\Delta$ is $\mathbb{Q}$-Cartier.

Given a variety $X$ and a Weil divisor $D$ on $X$, we say that $f: Y \rightarrow X$ is a log resolution of $(X, D)$ if $Y$ is regular, $\operatorname{Exc}(f)$ has pure codimension one, and $\left(Y, \operatorname{Supp}\left(f^{-1}(D)+\operatorname{Exc}(f)\right)\right)$ has simple normal crossings (see [Kol13, Definitions 1.8 and 1.12]). If $X$ is of dimension at most three, then a log resolution of $(X, D)$ always exists (see [Lip78, Cut09, CP08, CP09]).

Theorem 2.1 ([Kol13, Theorem 10.4]). Let $X$ be a regular surface over a field $k$, and let $f: X \rightarrow Y$ be a proper, generically finite morphism with exceptional curves $C_{i}$ such that $\bigcup_{i} C_{i}$ is connected. Let $L$ be a line bundle on $X$, and assume that there exist $\mathbb{Q}$-divisors $N$ and $\Delta=\sum d_{i} C_{i}$ such that

- $L \equiv_{f} K_{X}+\Delta+N$,

- $N \cdot C_{i} \geqslant 0$ for every $i$,

- $0 \leqslant d_{i}<1$ for every $i$.

Then $R^{1} f_{*} L=0$.

\subsection{Rational and Cohen-Macaulay singularities}

We say that a variety $X$ has rational singularities if it is Cohen-Macaulay and there exists a $\log$ resolution $f: Y \rightarrow X$ such that $R f_{*} \mathcal{O}_{Y}=\mathcal{O}_{X}$. If we assume the existence of resolutions of singularities, the equality holds for every log resolution (this follows from the main result 


\section{ON THE RATIONALITY OF KLT SINGULARITIES}

in [CR15]). For the definition of Serre's condition $S_{d}$ and Cohen-Macaulay singularities, we refer to [Kol13, Definitions 5.1 and 5.2].

Proposition 2.2. Let $f: Y \rightarrow X$ be a proper birational morphism of normal varieties, and let $D$ be a Weil divisor on $Y$ such that $\mathcal{O}_{Y}(D)$ is Cohen-Macaulay. Assume that $R^{i} f_{*} \mathcal{O}_{Y}(D)=0$ and $R^{i} f_{*} \mathcal{O}_{Y}\left(K_{Y}-D\right)=0$ for all $i>0$. Then $f_{*} \mathcal{O}_{Y}(D)$ is Cohen-Macaulay.

Proof. Since $D$ is Cohen-Macaulay, so is $\mathcal{H} \mathrm{m}_{\mathcal{O}_{Y}}\left(\mathcal{O}_{Y}(D), \omega_{Y}\right)$ (see [KM98, Corollary 5.70]). In particular, the latter sheaf is reflexive, and hence it is isomorphic to $\mathcal{O}_{Y}\left(K_{Y}-D\right)$. Therefore, the proposition follows from [Kol13, Theorem 2.74].

The following result is well known.

Proposition 2.3 ([Kov17, Lemma 8.1]). Let $X$ be a variety with rational singularities. Then $R f_{*} \omega_{Y}=\omega_{X}$ for every resolution $f: Y \rightarrow X$.

Let us recall that a rank one sheaf $\mathcal{F}$ on a normal variety $X$ is reflexive if and only if it is divisorial, that is, of the form $\mathcal{O}_{X}(D)$ for some Weil divisor $D$. Moreover, $i_{*}\left(\left.\mathcal{F}\right|_{U}\right) \simeq \mathcal{F}$, where $i: U \rightarrow X$ is an inclusion of an open subset whose complement is of codimension at least two.

Lemma 2.4. Let $X$ be a normal variety, let $S \subset X$ be a prime divisor, and let $D$ be any Weil divisor. For the exact sequence

$$
0 \rightarrow \mathcal{O}_{X}(-S+D) \rightarrow \mathcal{O}_{X}(D) \rightarrow \mathcal{E} \rightarrow 0
$$

we have that $\operatorname{Supp} \mathcal{E}=S$. Moreover, if $\mathcal{O}_{X}(-S+D)$ and $\mathcal{O}_{X}(D)$ satisfy Serre's condition $S_{3}$, then $\mathcal{E}$ is reflexive as a sheaf on $S$.

Proof. Since the ideal sheaf $\mathcal{I}_{S}$ of $S$ annihilates $\mathcal{E}$ and the sequence above is the standard restriction exact sequence outside of a closed subset of codimension two, we have that $\operatorname{Supp}(\mathcal{E})=S$. The second part of the lemma follows from [Kol13, Lemma 2.60].

\subsection{Strongly F-regular singularities}

The proof of the main theorem makes use of the concept of F-regular singularities. We refer the reader to [ST12] for a comprehensive treatment of this topic. The sole purpose of introducing them here is to show, summing up known results, that a plt threefold singularity $(X, S)$ in characteristic $p>5$ is rational near $S$ and, assuming $\mathbb{Q}$-factoriality, that any reflexive rank one sheaf on $X$ is Cohen-Macaulay near $S$ (Propositions 2.7 and 2.8, and, for the non- $\mathbb{Q}$-factorial case, Proposition 2.9). This shows that singularities of plt blow-ups possess these desirable properties (see Propositions 2.11 and 2.12). For the convenience of the reader, we recall the basic definitions.

Definition 2.5. For an F-finite scheme $\mathrm{X}$ of characteristic $p>0$ and an effective $\mathbb{Q}$-divisor $\Delta$, we say that $(X, \Delta)$ is globally $F$-split if for every $e \in \mathbb{Z}_{>0}$, the natural morphism

$$
\mathcal{O}_{X} \rightarrow F_{*}^{e} \mathcal{O}_{X}\left(\left\lfloor\left(p^{e}-1\right) \Delta\right\rfloor\right)
$$

splits in the category of sheaves of $\mathcal{O}_{X}$-modules.

We say that $(X, \Delta)$ is globally F-regular if for every effective divisor $D$ on $X$ and every big enough $e \in \mathbb{Z}_{>0}$, the natural morphism

$$
\mathcal{O}_{X} \rightarrow F_{*}^{e} \mathcal{O}_{X}\left(\left\lfloor\left(p^{e}-1\right) \Delta\right\rfloor+D\right)
$$

splits. 


\section{HACON AND J. WitASZEK}

We say that $(X, \Delta)$ is purely globally F-regular if the definition above holds for those $D$ which intersect $\lfloor\Delta\rfloor$ properly (see [Das15, Definition 2.3(2)]).

The local versions of the above notions are called F-purity, strong F-regularity, and pure F-regularity, respectively. Moreover, given a morphism $f: X \rightarrow Y$, we say that $(X, \Delta)$ is F-split, F-regular, or purely F-regular over $Y$ if the corresponding splittings hold locally over $Y$ (see [HX15, Definition 2.6]).

Remark 2.6. Assume that $(X, \Delta)$ is F-split over $Y$ with respect to $f: X \rightarrow Y$. Then for any affine open subset $U \subseteq Y$, we have that $\left(f^{-1}(U),\left.\Delta\right|_{f^{-1}(U)}\right)$ is globally F-split (see [HX15, Proposition 2.10], cf. [CGS16, Remark 2.8]). In particular, if $u: Y \rightarrow Y^{\prime}$ is affine, then $(X, \Delta)$ is also F-split over $Y^{\prime}$ with respect to $u \circ f$. Analogous statements hold for F-regularity and pure F-regularity.

Proposition 2.7. Let $X$ be a strongly F-regular variety. Then $X$ has rational singularities. Moreover, if $X$ is $\mathbb{Q}$-factorial and of dimension at least three, then any reflexive sheaf of rank one on it is $S_{3}$.

In particular, a reflexive sheaf of rank one on a $\mathbb{Q}$-factorial strongly F-regular threefold is Cohen-Macaulay.

Proof. The variety $X$ is F-rational (see [ST12, Appendix C]), and hence it has rational singularities by [Kov17, Corollary 1.12].

The last statement in the proposition is a consequence of [PS14, Theorem 3.8]. Indeed, let $\mathcal{O}_{X}(D)$ be a reflexive sheaf, and take a general effective divisor $E \sim-(m p+1) D$ for $m \gg 0$. Then $(X,(1 /(m p+1)) E)$ is strongly F-regular, and the result follows.

The following proposition is false in characteristic $p=2$ (see [CT16]).

Proposition 2.8. Let $(X, S)$ be a plt three-dimensional pair defined over an algebraically closed field of characteristic $p>5$. Then $S$ is normal, and $X$ is strongly F-regular along it.

Proof. By [Har98] and [Das15, Theorem A] (cf. Lemma 2.10), we have that $(X, S)$ is purely $F$-regular along $S$ and $S$ is normal. This implies the F-regularity of $X$ along $S$.

In order to deal with non- $\mathbb{Q}$-factorial singularities, we also need a relative version of the above result.

Proposition 2.9. Let $(X, S)$ be a plt three-dimensional pair defined over an algebraically closed field of characteristic $p>5$, and let $f: X \rightarrow Z$ be a proper birational morphism between normal varieties such that $\operatorname{dim} f(S)=2$. If $-\left(K_{X}+S\right)$ is $f$-ample, then $Z$ is strongly F-regular along $f(S)$.

This does not follow directly from Proposition 2.8, because $K_{Z}+f(S)$ is almost never $\mathbb{Q}$ Cartier. Further, let us note that we will need to apply Proposition 2.9 in the case when $X$ is not $\mathbb{Q}$-factorial.

Proof. As above, $S$ is normal. The normalization morphism $f(S)^{\nu} \rightarrow f(S)$ is affine, and so, by [HX15, Theorem 3.1], the pair $\left(S, \Delta_{\text {diff }}\right)$, defined by adjunction, $K_{S}+\Delta_{\text {diff }}=\left.\left(K_{X}+S\right)\right|_{S}$, is globally $F$-regular over $f(S)$ (see Remark 2.6). Lemma 2.10 and [HX15, Lemma 2.12] imply that $Z$ is strongly $F$-regular along $f(S)$. 


\section{ON THE RATIONALITY OF KLT SINGULARITIES}

In Proposition 2.9, we used a relative version of inversion of adjunction, which is a consequence of [Das15, Theorem B]. The proof is very similar to [Das15, Corollary 5.4] and [CTW17, Lemma 2.7], but we include it here for the convenience of the reader.

Lemma 2.10. Let $(X, S+B)$ be a plt pair where $S$ is a prime divisor, and let $f: X \rightarrow Z$ be a proper birational morphism between normal varieties. Assume that $-\left(K_{X}+S+B\right)$ is $f$-ample and $\left(\bar{S}, B_{\bar{S}}\right)$ is globally $F$-regular over $f(S)$, where $\bar{S}$ is the normalization of $S$, and $B_{\bar{S}}$ is defined by adjunction, $K_{\bar{S}}+B_{\bar{S}}=\left.\left(K_{X}+S+B\right)\right|_{\bar{S}}$. Then $(X, S+B)$ is purely globally F-regular over a Zariski-open neighbourhood of $f(S) \subseteq Z$.

Proof. Since the question is local over $Z$, we can assume that $Z$ is affine, that is, $Z=\operatorname{Spec}(R)$ for some ring $R$. Further, by perturbing the coefficients of $B$, we can assume that the Cartier index of $K_{X}+S+B$ is not divisible by $p$ (see, for example, the proof of [Das15, Proposition 3.8]). By [Das15, Theorem A], the pair $(X, S+B)$ is purely F-regular, and $S$ is a normal F-pure centre. By standard arguments (replacing $B$ with $B+\epsilon H$ for $0<\epsilon \ll 1$ and a very ample divisor $H$ intersecting $S$ properly), it is enough to show that $(X, S+B)$ is $F$-split over a Zariski-open neighbourhood of $f(S)$ (see the proof of [SS10, Theorem 3.9]).

Set $\mathcal{L}:=\mathcal{O}_{X}\left(\left(1-p^{e}\right)\left(K_{X}+S+B\right)\right)$, and for a sufficiently divisible integer $e \gg 0$, consider the diagram

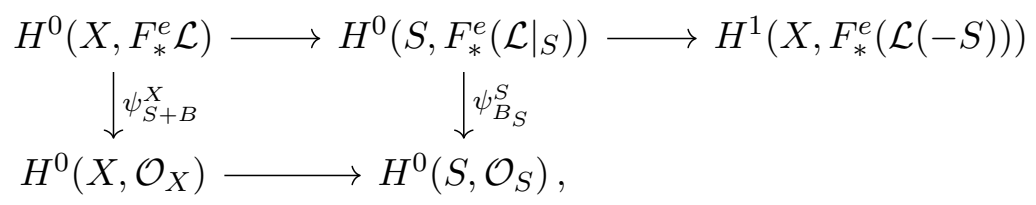

where $\psi_{S+B}^{X}$ and $\psi_{B_{S}}^{S}$ are the trace maps, while the horizontal arrows come from the restriction exact sequences. The diagram is well defined and commutes by the fact that $S$ is an F-pure centre and by the equality of the different and the F-different (see [Das15, Theorem B], cf. [CTW17, Section 2.2]). Note that $\psi_{B_{S}}^{S}$ is surjective since $\left(S, B_{S}\right)$ is F-split over $f(S)$.

Since $e \gg 0$, the relative Serre vanishing theorem implies that $H^{1}\left(X, F_{*}^{e}(\mathcal{L}(-S))\right)=0$. In particular, the upper left horizontal arrow is surjective, and so the composition

$$
H^{0}\left(X, F_{*}^{e} \mathcal{L}\right) \stackrel{\psi_{S+B}^{X}}{\longrightarrow} H^{0}\left(X, \mathcal{O}_{X}\right) \rightarrow H^{0}\left(S, \mathcal{O}_{S}\right)
$$

is surjective as well. But $H^{0}\left(X, \mathcal{O}_{X}\right)=H^{0}\left(Z, \mathcal{O}_{Z}\right)=R$; hence, this implies that the zero locus of the ideal in $R$ generated by $\operatorname{im}\left(\psi_{S+B}^{X}\right)$ is disjoint from $f(S)$. After we remove this locus, the surjectivity of $\psi_{S+B}^{X}$ follows.

\subsection{Local to global transition}

In the course of the proof of the main theorem, we will need the following two results that allow for the transition from a local to a global case.

Proposition 2.11 (Generalized plt blow-up, Kollár component). Let $(X, \Delta)$ be a Kawamata $\log$ terminal three-dimensional pair defined over an algebraically closed field of characteristic $p>5$, and let $x \in X$ be a closed point. Assume that $X \backslash\{x\}$ is $\mathbb{Q}$-factorial. Then there exist a projective birational morphism $f: Y \rightarrow X$ and an effective $\mathbb{Q}$-divisor $\Delta_{Y}$ on $Y$ such that

- $f$ is an isomorphism over $X \backslash\{x\}$,

- $S:=\operatorname{Exc} f$ is irreducible and anti- $f$-nef, 


\section{HACON AND J. WitASZEK}

- $\left(Y, S+\Delta_{Y}\right)$ is a $\mathbb{Q}$-factorial plt threefold,

- $-\left(K_{Y}+S+\Delta_{Y}\right)$ is $f$-ample.

Furthermore, $S$ is normal, and $Y$ is strongly F-regular along it.

When $x \in X$ is $\mathbb{Q}$-factorial, $-S$ is automatically $f$-ample. Since $X$ is a klt threefold, there exists an open neighbourhood $x \in U \subseteq X$ such that $U \backslash\{x\}$ is $\mathbb{Q}$-factorial.

Proof. Apart from the last statement, this is a direct consequence of [GNT15, Proposition 2.15] (see [Xu14, Lemma 1]). The strong F-regularity of $Y$ and the normality of $S$ follow from Proposition 2.8 .

For the non- $\mathbb{Q}$-factorial case of the main theorem, we will also need the following result.

Proposition 2.12. Under the assumptions of Proposition 2.11, the f-relative semiample fibration $g: Y \rightarrow Y^{\prime}$ associated with $-S$ exists. Moreover, $g$ is small, $-\left.S\right|_{S}$ is big, and $Y^{\prime}$ is strongly F-regular along $g(S)$.

Proof. The first statement follows from the base point free theorem (see [GNT15, Theorem 2.9]), once we notice that $-S=K_{Y}+\Delta_{Y}-\left(K_{Y}+S+\Delta_{Y}\right)$.

For the proof of the bigness of $-\left.S\right|_{S}$, it is enough to show that $g$ is small. To this end, run a $\left(K_{Y}+S+\Delta_{Y}\right)$-MMP over $Y^{\prime}$, and let $Y \rightarrow Y_{\min } \stackrel{g^{\prime}}{\rightarrow} Y^{\prime}$ be the minimal model. Since $-\left(K_{Y}+S+\Delta_{Y}\right)$ is $g$-ample, $g^{\prime}$ is small. Moreover, as $S$ is $g$-numerically trivial, none of the steps of the MMP may contract $S$. Indeed, an MMP preserves $\mathbb{Q}$-factoriality, and hence a contracted divisor is always relatively anti-ample with respect to a divisorial contraction. Since Exc $f=S$ is irreducible, this shows that $g$ is small and does not contract $S$.

For the last statement, we replace $Y$ with the output of a $-\left(K_{Y}+S\right)$-MMP over $Y^{\prime}$, and so we can assume that $-\left(K_{Y}+S\right)$ is $g$-nef and $g$-big. We can run such an MMP, since we can write

$$
-\epsilon\left(K_{Y}+S\right)=K_{Y}+S+(1+\epsilon) \Delta_{Y}-(1+\epsilon)\left(K_{Y}+S+\Delta_{Y}\right),
$$

where $-\left(K_{Y}+S+\Delta_{Y}\right)$ is $g$-ample and $\left(Y, S+(1+\epsilon) \Delta_{Y}\right)$ is plt for $0<\epsilon \ll 1$. Further, by replacing $Y$ with the image of the $g$-relative semiample fibration associated with $-\left(K_{Y}+S\right)$, we can assume that $-\left(K_{Y}+S\right)$ is $g$-ample. The fibration exists by the klt base point free theorem, as $S$ is $g$-numerically trivial and we can write

$$
-\left(K_{Y}+S\right)=K_{Y}-2\left(K_{Y}+S\right)+S .
$$

Although $Y$ may have ceased to be $\mathbb{Q}$-factorial, $(Y, S)$ is a well-defined plt pair (in particular, $K_{Y}+S$ is $\mathbb{Q}$-Cartier), and so Proposition 2.9 concludes the proof.

\subsection{Liftings}

Let $W_{m}(k)$ denote the ring of Witt vectors of length $m$. We say that a $k$-variety $X$ lifts over $W_{m}(k)$ if there exists a flat morphism $\widetilde{X} \rightarrow \operatorname{Spec} W_{m}(k)$ such that the special fibre is isomorphic to $X$. Similarly, we can define liftings of morphisms.

In Section 5, we will need the following result.

Proposition 2.13 ([LS14, Proposition 2.1], [CvS09, Theorem 3.1]). Let $f: \underset{\widetilde{Y}}{Y} \rightarrow X$ be a morphism of schemes satisfying $R f_{*} \mathcal{O}_{Y}=\mathcal{O}_{X}$. Assume that $Y$ lifts to a scheme $\widetilde{Y}_{m}$ over $W_{m}(k)$ for some natural number $m \in \mathbb{Z}_{>0}$. Then $f$ lifts to $\widetilde{f}_{m}: \widetilde{Y}_{m} \rightarrow \widetilde{X}_{m}$ over $W_{m}(k)$.

The same is true for formal lifts to the total Witt ring $W(k)$. 


\section{ON THE RATIONALITY OF KLT SINGULARITIES}

\section{The restriction short exact sequence}

Proposition 3.1. Let $(X, S)$ be a plt three-dimensional $\mathbb{Q}$-factorial pair defined over an algebraically closed field $k$ of characteristic $p>5$, where $S$ is a prime divisor, and let $\Delta_{\text {diff }}$ be the different. Then for any Weil divisor $D$ on $X$, there exist an effective $\mathbb{Q}$-divisor $\Delta_{S} \leqslant \Delta_{\text {diff }}$ and a Weil divisor $G$ on $S$ such that $G \sim_{\mathbb{Q}} K_{S}+\Delta_{S}+\left.D\right|_{S}$ and the sequence

$$
0 \rightarrow \mathcal{O}_{X}\left(K_{X}+D\right) \rightarrow \mathcal{O}_{X}\left(K_{X}+S+D\right) \rightarrow \mathcal{O}_{S}(G) \rightarrow 0
$$

is exact.

Let us note that $G$ is only defined up to linear equivalence.

Proof. Note that in proving the proposition, we are free to replace $X$ with an appropriate neighbourhood of $S$. By Proposition 2.8, we may assume that $X$ is strongly F-regular and $S$ is normal. Replacing $D$ with a linearly equivalent divisor, we may assume that $S$ is not contained in the support of $D$ and so $\left.D\right|_{S}$ is a well-defined $\mathbb{Q}$-divisor. Consider the following short exact sequence (see Lemma 2.4):

$$
0 \rightarrow \mathcal{O}_{X}\left(K_{X}+D\right) \rightarrow \mathcal{O}_{X}\left(K_{X}+S+D\right) \rightarrow i_{*} \mathcal{E} \rightarrow 0
$$

where $\mathcal{E}$ is a sheaf supported on $S$ and $i: S \rightarrow X$ is the inclusion. By Proposition 2.7, any reflexive rank one sheaf on $X$ satisfies Serre's condition $S_{3}$ and so, by Lemma 2.4, the sheaf $\mathcal{E}$ is reflexive on $S$.

Let $\pi: \bar{X} \rightarrow X$ be a $\log$ resolution of $(X, S)$, and let $\bar{S}$ be the strict transform of $S$. Define $\Delta_{\bar{X}}$ by

$$
K_{\bar{X}}+\bar{S}+\Delta_{\bar{X}}=\pi^{*}\left(K_{X}+S\right)
$$

Consider the following exact sequence:

$$
0 \rightarrow \mathcal{O}_{\bar{X}}\left(K_{\bar{X}}+\left\lceil\pi^{*} D\right\rceil\right) \rightarrow \mathcal{O}_{\bar{X}}\left(K_{\bar{X}}+\bar{S}+\left\lceil\pi^{*} D\right\rceil\right) \rightarrow \mathcal{O}_{\bar{S}}\left(K_{\bar{S}}+\left.\left\lceil\pi^{*} D\right\rceil\right|_{\bar{S}}\right) \rightarrow 0 .
$$

Since $(X, S)$ is plt, we have that $\left\lfloor\Delta_{\bar{X}}\right\rfloor \leqslant 0$, and so $\left\lceil\pi^{*} D\right\rceil \geqslant\left\lfloor\Delta_{\bar{X}}+\pi^{*} D\right\rfloor$. Therefore,

$$
\begin{aligned}
K_{\bar{X}}+\bar{S}+\left\lceil\pi^{*} D\right\rceil & \geqslant\left\lfloor K_{\bar{X}}+\bar{S}+\Delta_{\bar{X}}+\pi^{*} D\right\rfloor \\
& =\left\lfloor\pi^{*}\left(K_{X}+S+D\right)\right\rfloor, \quad \text { and } \\
K_{\bar{X}}+\left\lceil\pi^{*} D\right\rceil & \geqslant\left\lfloor K_{\bar{X}}+\Delta_{\bar{X}}+\pi^{*} D\right\rfloor \\
& \geqslant\left\lfloor\pi^{*}\left(K_{X}+D\right)\right\rfloor .
\end{aligned}
$$

In particular,

$$
\begin{aligned}
\pi_{*} \mathcal{O}_{\bar{X}}\left(K_{\bar{X}}+\left\lceil\pi^{*} D\right\rceil\right) & =\mathcal{O}_{X}\left(K_{X}+D\right), \quad \text { and } \\
\pi_{*} \mathcal{O}_{\bar{X}}\left(K_{\bar{X}}+\bar{S}+\left\lceil\pi^{*} D\right\rceil\right) & =\mathcal{O}_{X}\left(K_{X}+S+D\right) .
\end{aligned}
$$

By applying $\pi_{*}$ to the above exact sequence, we have that

$$
0 \rightarrow \mathcal{O}_{X}\left(K_{X}+D\right) \rightarrow \mathcal{O}_{X}\left(K_{X}+S+D\right) \rightarrow \pi_{*} \mathcal{O}_{\bar{S}}(\bar{G}) \rightarrow R^{1} \pi_{*} \mathcal{O}_{\bar{X}}\left(K_{\bar{X}}+\left\lceil\pi^{*} D\right\rceil\right)
$$

is exact, where $\bar{G}:=K_{\bar{S}}+\left.\left\lceil\pi^{*} D\right\rceil\right|_{\bar{S}}$. By localizing at one-dimensional points on $X$, we can use Lemma 3.3 to show that

$$
\operatorname{dim} \operatorname{Supp}\left(R^{1} \pi_{*} \mathcal{O}_{\bar{X}}\left(K_{\bar{X}}+\left\lceil\pi^{*} D\right\rceil\right)\right) \leqslant 0 .
$$

Set $\Delta_{S}:=\left(\left.\pi\right|_{\bar{S}}\right)_{*}\left(\left.\left(\left\lceil\pi^{*} D\right\rceil-\pi^{*} D\right)\right|_{\bar{S}}\right)$ and $G:=\left(\left.\pi\right|_{\bar{S}}\right)_{*} \bar{G}$, so that $G \sim_{\mathbb{Q}} K_{S}+\Delta_{S}+\left.D\right|_{S}$. We claim that $\mathcal{E} \simeq \mathcal{O}_{S}(G)$. Indeed, by what we observed above, $\mathcal{E}$ is isomorphic to $\pi_{*} \mathcal{O}_{\bar{S}}(\bar{G})$ in 


\section{HACON AND J. WitASZEK}

codimension one on $S$, which in turn is isomorphic to $\mathcal{O}_{S}(G)$ in codimension one on $S$. Since both $\mathcal{E}$ and $\mathcal{O}_{S}(G)$ are reflexive sheaves of rank one, the isomorphism $\mathcal{E} \simeq \mathcal{O}_{S}(G)$ follows.

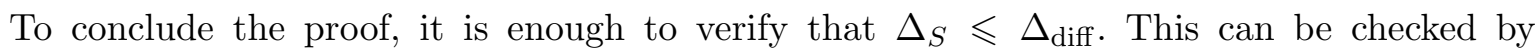
localizing at one-dimensional points in $\operatorname{Sing}(X)$, and thus we conclude by Lemma 3.3 (see also [Kol13, Theorem 3.36] and [Kol92, Corti 16.6.3]).

Remark 3.2. Proposition 3.1 also holds in the following two cases:

(1) if $(X, S)$ is an $n$-dimensional $\mathbb{Q}$-factorial plt pair and $D$ is a Weil divisor, defined over an algebraically closed field of characteristic 0 , and

(2) if $(X, S)$ is an $n$-dimensional $\mathbb{Q}$-factorial plt pair and $D$ is a Weil divisor, defined over an algebraically closed field of characteristic $p>0$ such that $(X, S)$ admits a log resolution of singularities and $\left(S^{\nu}, \Delta_{\text {diff }}\right)$ is strongly F-regular, where $S^{\nu} \rightarrow S$ is the normalization.

For case $(1)$, recall that since $(X, S)$ is plt, $S$ is normal and both $\mathcal{O}_{X}\left(K_{X}+D\right)$ and $\mathcal{O}_{X}\left(K_{X}+\right.$ $S+D)$ are Cohen-Macaulay sheaves by [KM98, Corollary 5.25]. For case (2), recall that by [Das15], the divisor $S$ is normal and $(X, S)$ is purely F-regular near $S$.

The following lemma, used in the above proof, was applied to localizations at non-closed points, and thus we cannot assume that $k$ is algebraically closed. However, this has no impact on the proof because the surfaces under consideration are excellent. We refer to [Kol13] and [Tan18] for the classification and basic results pertaining to excellent log canonical surfaces. Let us just note that excellent klt surfaces are $\mathbb{Q}$-factorial by [Tan18, Corollary 4.10], and if $(X, S)$ is a plt surface pair, then $S$ is regular [Kol13, Section 3.35].

Lemma 3.3. Let $(X, S)$ be a plt surface pair defined over an arbitrary field $k$, where $S$ is a prime divisor. Let $\Delta_{\text {diff }}$ be the different, let $\pi: \bar{X} \rightarrow X$ be a $\log$ resolution of $(X, S)$, let $\bar{S}$ be the strict transform of $S$, and let $D$ be any Weil divisor. Then

- $R^{1} \pi_{*} \mathcal{O}_{\bar{X}}\left(K_{\bar{X}}+\left\lceil\pi^{*} D\right\rceil\right)=0$, and

- $\Delta_{S} \leqslant \Delta_{\text {diff }}$, where $\Delta_{S}:=\left.\left(\left\lceil\pi^{*} D\right\rceil-\pi^{*} D\right)\right|_{\bar{S}}$ and $S$ is identified with $\bar{S}$.

Proof. As $\left\lfloor\left\lceil\pi^{*} D\right\rceil-\pi^{*} D\right\rfloor=0$, the vanishing $R^{1} \pi_{*} \mathcal{O}_{\bar{X}}\left(K_{\bar{X}}+\left\lceil\pi^{*} D\right\rceil\right)=0$ follows by Theorem 2.1.

As for the second statement, we can restrict ourselves to a neighbourhood of $S$ such that all singularities of $X$ lie on $S$. Therefore, [Kol13, Section 3.35] implies that each singularity of $X$ is cyclic and $S$ is regular. Moreover, if $x \in \operatorname{Sing}(X)$ and $m_{x}$ is the $\mathbb{Q}$-factorial index of $X$ at $x$, then $m_{x} D$ is Cartier and $\Delta_{\text {diff }}$ has coefficient $1-1 / m_{x}$ at $x$ [Kol92, Corti 16.6.3]. Therefore, $\Delta_{S} \leqslant \Delta_{\text {diff }}$ because, by definition, $\Delta_{S}$ has coefficients smaller than one, and $m_{x} \Delta_{S}$ is Cartier at $x \in \operatorname{Sing}(X)$.

By applying Proposition 3.1 for $D$ replaced with $D-\left(K_{X}+S\right)$, we get that

$$
0 \rightarrow \mathcal{O}_{X}(-S+D) \rightarrow \mathcal{O}_{X}(D) \rightarrow \mathcal{O}_{S}(G) \rightarrow 0
$$

is exact for a $\mathbb{Q}$-divisor $0 \leqslant \Delta_{S} \leqslant \Delta_{\text {diff }}$ and a Weil divisor $\left.G \sim_{\mathbb{Q}} D\right|_{S}-\Delta_{S}$.

Corollary 3.4. Under the assumptions of Proposition 3.1, the sequences

$$
\begin{aligned}
& 0 \rightarrow \mathcal{O}_{X}\left(K_{X}-(k+1) S-D\right) \rightarrow \mathcal{O}_{X}\left(K_{X}-k S-D\right) \rightarrow \mathcal{O}_{S}\left(G_{k}^{\prime}\right) \rightarrow 0, \\
& 0 \rightarrow \mathcal{O}_{X}(-(k+1) S+D) \rightarrow \mathcal{O}_{X}(-k S+D) \rightarrow \mathcal{O}_{S}\left(G_{k}\right) \rightarrow 0
\end{aligned}
$$

are exact for any $k \in \mathbb{Z}_{>0}$, some effective $\mathbb{Q}$-divisors $\Delta_{k}, \Delta_{k}^{\prime} \leqslant \Delta_{\text {diff }}$ depending on $k$, and some integral divisors $G_{k}^{\prime} \sim_{\mathbb{Q}} K_{S}+\Delta_{k}^{\prime}-\left.(k+1) S\right|_{S}-\left.D\right|_{S}$ and $G_{k} \sim_{\mathbb{Q}}-\left.k S\right|_{S}+\left.D\right|_{S}-\Delta_{k}$. 


\section{ON THE RATIONALITY OF KLT SINGULARITIES}

\section{Proof of the main theorem}

Proof of Theorem 1.1. Fix $p_{0}$ as in Theorem 1.2. Since the question is local, we can assume that $X$ is affine and aim to show that a fixed closed point $x \in X$ is rational. We replace $X$ with a Zariski-open neighbourhood of $x$ whenever convenient. Let $f: Y \rightarrow X$ be a generalized plt blow-up at $x$ as in Proposition 2.11. Let $S:=\operatorname{Exc}(f)$. Recall that $\left(Y, S+\Delta_{Y}\right)$ is plt, $-S$ is $f$-nef, and $-\left(K_{Y}+S+\Delta_{Y}\right)$ is $f$-ample for some effective $\mathbb{Q}$-divisor $\Delta_{Y}$ on $Y$. Moreover, we may assume that $Y$ is strongly F-regular. First, we show that $R f_{*} \mathcal{O}_{Y}=\mathcal{O}_{X}$.

To this end, we apply Corollary 3.4 to get short exact sequences

$$
0 \rightarrow \mathcal{O}_{Y}(-(k+1) S) \rightarrow \mathcal{O}_{Y}(-k S) \rightarrow \mathcal{O}_{S}\left(G_{k}\right) \rightarrow 0
$$

for all $k \geqslant 0$, where $G_{k} \sim_{\mathbb{Q}}-\left.k S\right|_{S}-\Delta_{k}$ for some effective $\mathbb{Q}$-divisor $\Delta_{k} \leqslant \Delta_{\text {diff }}$, where $\Delta_{\text {diff }}$ is the different. Thus, to show that $R^{i} f_{*} \mathcal{O}_{Y}(-k S)$ vanishes for $k=0$ and $i>0$, it is enough to prove that

- $R^{i} f_{*} \mathcal{O}_{Y}(-m S)=0$ for $i>0$ and a divisible enough $m \gg 0$, and

- $H^{i}\left(S, \mathcal{O}_{S}\left(G_{k}\right)\right)=0$ for $i>0$ and all $k \geqslant 0$.

If $X$ is $\mathbb{Q}$-factorial, then the first vanishing follows from the relative Serre vanishing theorem, as $-S$ is $f$-ample. In general, we consider the $f$-relative semiample fibration $Y \stackrel{g}{\rightarrow} Y^{\prime} \stackrel{f^{\prime}}{\rightarrow} X$ associated with $-S$. It exists by Proposition 2.12. Since $Y^{\prime}$ is strongly F-regular along $S_{Y^{\prime}}:=$ $g(S)$, we may assume that $Y^{\prime}$ has rational singularities (see Proposition 2.7). For $m>0$ sufficiently divisible, $m S_{Y^{\prime}}$ is Cartier and $m S=g^{*}\left(m S_{Y^{\prime}}\right)$. By the projection formula, we have that $R g_{*} \mathcal{O}_{Y}(-m S)=g_{*} \mathcal{O}_{Y}(-m S)=\mathcal{O}_{Y^{\prime}}\left(-m S_{Y^{\prime}}\right)$ and therefore $R^{i} f_{*} \mathcal{O}_{Y}(-m S)=R^{i} f_{*}^{\prime} \mathcal{O}_{Y^{\prime}}\left(-m S_{Y^{\prime}}\right)$, which is zero for $m \gg 0$ by the Serre vanishing theorem since $S_{Y^{\prime}}$ is $f^{\prime}$-anti-ample.

Therefore, it suffices to show that $H^{i}\left(S, \mathcal{O}_{S}\left(G_{k}\right)\right)=0$ for $i>0$ and all $k \geqslant 0$. To this end, we notice that

$$
\left.\left(K_{Y}+S+\Delta_{Y}\right)\right|_{S}=K_{S}+\Delta_{\text {diff }}+\left.\Delta_{Y}\right|_{S}
$$

is anti-ample and $\left(S, \Delta_{\text {diff }}+\left.\Delta_{Y}\right|_{S}\right)$ is klt. The cohomology in question vanishes because

$$
G_{k} \sim_{\mathbb{Q}} K_{S}+\left(\Delta_{\text {diff }}+\left.\Delta_{Y}\right|_{S}-\Delta_{k}\right) \underbrace{-\left(K_{S}+\Delta_{\text {diff }}+\left.\Delta_{Y}\right|_{S}\right)-\left.k S\right|_{S}}_{\text {ample }},
$$

which is zero by Theorem 1.2.

By an analogous argument, considering the other short exact sequence in Corollary 3.4,

$$
0 \rightarrow \mathcal{O}_{Y}\left(K_{Y}-(k+1) S\right) \rightarrow \mathcal{O}_{Y}\left(K_{Y}-k S\right) \rightarrow \mathcal{O}_{S}\left(G_{k}^{\prime}\right) \rightarrow 0
$$

one can show that $R^{i} f_{*} \omega_{Y}=0$ for $i>0$. Indeed, $R g_{*} \omega_{Y}=\omega_{Y^{\prime}}$ by Proposition 2.3 applied to both $Y$ and $Y^{\prime}$. As $S$ is $g$-trivial, we have that

$$
R^{i} f_{*} \mathcal{O}_{Y}\left(K_{Y}-m S\right)=R^{i} f_{*}^{\prime} \mathcal{O}_{Y^{\prime}}\left(K_{Y^{\prime}}-m S_{Y^{\prime}}\right)=0
$$

for $i>0$ and a divisible enough $m \gg 0$ by the relative Serre vanishing theorem. Moreover, by Theorem 1.2,

$$
H^{i}\left(S, \mathcal{O}_{S}\left(G_{k}^{\prime}\right)\right)=0 \quad \forall i>0
$$

for any $k \geqslant 0$ and a divisor $G_{k}^{\prime}$ such that $G_{k}^{\prime} \sim_{\mathbb{Q}} K_{S}+\Delta_{k}^{\prime}-\left.(k+1) S\right|_{S}$, where $\Delta_{k}^{\prime}$ is an effective $\mathbb{Q}$-divisor satisfying $\Delta_{k}^{\prime} \leqslant \Delta_{\text {diff }}$, because $-\left.S\right|_{S}$ is nef and big (see Proposition 2.12).

Proposition 2.7 implies that $Y$ is Cohen-Macaulay, and so $X$ is Cohen-Macaulay as well by Proposition 2.2. Let $\pi_{Y}: \bar{Y} \rightarrow Y$ be a $\log$ resolution. By Proposition 2.7, we have that 


\section{Hacon AND J. WitAsZeK}

$R\left(\pi_{Y}\right)_{*} \mathcal{O}_{\bar{Y}}=\mathcal{O}_{Y}$, and so, by the composition of derived functors, we get that $R \pi_{*} \mathcal{O}_{\bar{Y}}=\mathcal{O}_{X}$, where $\pi:=f \circ \pi_{Y}: \bar{Y} \rightarrow X$. Thus $X$ has rational singularities.

In order to prove the last statement, we assume that $X$ is $\mathbb{Q}$-factorial, fix a Weil divisor $D$ on $X$, and set $D_{Y}:=\left\lfloor f^{*} D\right\rfloor=f^{*} D-\epsilon S$ for some $0 \leqslant \epsilon<1$.

First, we show that $R f_{*} \mathcal{O}_{Y}\left(D_{Y}\right)=\mathcal{O}_{X}(D)$. By Corollary 3.4, we get short exact sequences

$$
0 \rightarrow \mathcal{O}_{Y}\left(-(k+1) S+D_{Y}\right) \rightarrow \mathcal{O}_{Y}\left(-k S+D_{Y}\right) \rightarrow \mathcal{O}_{S}\left(G_{k}\right) \rightarrow 0
$$

for all $k \geqslant 0$, where $G_{k} \sim_{\mathbb{Q}}-\left.(k+\epsilon) S\right|_{S}-\Delta_{k}$ for some effective $\mathbb{Q}$-divisor $\Delta_{k} \leqslant \Delta_{\text {diff. As in the }}$ argument above, when $i>0$ we have that

$$
\begin{aligned}
H^{i}\left(S, \mathcal{O}_{S}\left(G_{k}\right)\right)=0 & \text { and } \\
R^{i} f_{*} \mathcal{O}_{Y}\left(-m S+D_{Y}\right)=0 & \text { for divisible enough } m \gg 0,
\end{aligned}
$$

where the second identity follows from the Serre vanishing theorem as $-S$ is $f$-ample, and the first one is a consequence of Theorem 1.2 given that

$$
G_{k} \sim_{\mathbb{Q}} K_{S}+\left(\Delta_{\text {diff }}+\left.\Delta_{Y}\right|_{S}-\Delta_{k}\right) \underbrace{-\left(K_{S}+\Delta_{\text {diff }}+\left.\Delta_{Y}\right|_{S}\right)-\left.(k+\epsilon) S\right|_{S}}_{\text {ample }} .
$$

The claim now follows easily by an argument similar to the one we used in the proof that the singularities are rational.

Analogously, one can show that $R^{i} f_{*} \mathcal{O}_{Y}\left(K_{Y}-D_{Y}\right)=0$ for $i>0$ by considering the other short exact sequence in Corollary 3.4,

$$
0 \rightarrow \mathcal{O}_{Y}\left(K_{Y}-(k+1) S-D_{Y}\right) \rightarrow \mathcal{O}_{Y}\left(K_{Y}-k S-D_{Y}\right) \rightarrow \mathcal{O}_{S}\left(G_{k}^{\prime}\right) \rightarrow 0 .
$$

Indeed, $R^{i} f_{*} \mathcal{O}_{Y}\left(K_{Y}-m S-D_{Y}\right)=0$ for sufficiently divisible $m \gg 0$ by the Serre vanishing theorem. Moreover, by Theorem 1.2,

$$
H^{i}\left(S, \mathcal{O}_{S}\left(G_{k}^{\prime}\right)\right)=0 \quad \forall i>0
$$

for any $k \geqslant 0$ and a divisor $G_{k}^{\prime}$ such that $G_{k}^{\prime} \sim_{\mathbb{Q}} K_{S}+\Delta_{k}^{\prime}-\left.(k+1-\epsilon) S\right|_{S}$, where $\Delta_{k}^{\prime}$ is an effective $\mathbb{Q}$-divisor satisfying $\Delta_{k}^{\prime} \leqslant \Delta_{\text {diff }}$.

Since $\mathcal{O}_{Y}\left(D_{Y}\right)$ is Cohen-Macaulay (see Proposition 2.7), Proposition 2.2 concludes the proof.

\section{Applications and open problems}

Corollary 5.1. There exists a constant $p_{0}>0$ with the following property. Let $\left(X, \Delta_{X}\right)$ and $\left(Y, \Delta_{Y}\right)$ be Kawamata log terminal threefolds defined over an algebraically closed field $k$ of characteristic $p>p_{0}$, and let $\pi: X \rightarrow Y$ be a proper birational morphism between them. If $X$ lifts to $\widetilde{X}_{m}$ over $W_{m}(k)$ for some $m \in \mathbb{N}$, then $\pi$ lifts to $\widetilde{\pi}_{m}: \widetilde{X}_{m} \rightarrow \widetilde{Y}_{m}$.

Proof. This follows from Theorem 1.1 and Proposition 2.13.

With Corollary 5.1 in mind, it is natural to ask the following.

Question 5.2. Let $X$ be a Kawamata $\log$ terminal threefold defined over an algebraically closed field $k$ of characteristic $p \gg 0$ and $X \rightarrow X^{+}$a flip. If $X$ lifts to $\widetilde{X}_{m}$ over $W_{m}(k)$ for some $m \in \mathbb{N}$, then does $X^{+}$lift to $\widetilde{X}_{m}^{+}$over $W_{m}(k)$ ? 


\section{ON THE RATIONALITY OF KLT SINGULARITIES}

Remark 5.3. After the first version of the paper had been announced, we were informed by Professor Takehiko Yasuda that for any algebraically closed field $k$ of characteristic $p>2$, there exists a quotient klt singularity of dimension $d$ depending on $p$ which is not Cohen-Macaulay. For $p \geqslant 5$, his construction is the following. Let $X=V / G$, where $V$ is a $d$-dimensional $k$-vector space, $d(d-1) / 2 \geqslant p \geqslant d \geqslant 4$, and $G \simeq \mathbb{F}_{p}$ is a subgroup of $\mathrm{GL}_{d}(k)$ generated by a matrix of the form

$$
\left[\begin{array}{ccccccc}
1 & 1 & 0 & 0 & \ldots & 0 & 0 \\
0 & 1 & 1 & 0 & \ldots & 0 & 0 \\
0 & 0 & 1 & 1 & \ldots & 0 & 0 \\
0 & 0 & 0 & 1 & \ldots & 0 & 0 \\
& & & & \ddots & & \\
0 & 0 & 0 & 0 & \ldots & 1 & 1 \\
0 & 0 & 0 & 0 & \ldots & 0 & 1
\end{array}\right],
$$

that is, $\mathrm{Id}+I$. Here Id is the identity matrix and $I$ is a matrix with ones just above the diagonal and zeroes elsewhere. We have that $(\operatorname{Id}+I)^{p}=\operatorname{Id}$ because $p \geqslant d$. Since the action of $G$ on $V$ is indecomposable and $d(d-1) / 2 \geqslant p$, the variety $X$ is klt by [Yas14, Propositions 6.6 and 6.9]. Since $\operatorname{dim} V^{G}=1<d-2$, it is not Cohen-Macaulay by the main result in [ES80] (see the first paragraph of the article).

By a similar construction, one can get an example of a non-Cohen-Macaulay quotient klt singularity for $p=3$.

It is also natural to wonder what the optimal value for the constant $p_{0}$ is.

Question 5.4. Let $X$ be a three-dimensional klt variety defined over an algebraically closed field of characteristic $p>2$. Does $X$ have rational singularities?

\section{ACKNOWLEDGEMENTS}

We would like to thank Fabio Bernasconi, Paolo Cascini, János Kollár, Sándor Kovács, Karl Schwede, Hiromu Tanaka, and Takehiko Yasuda for comments and helpful suggestions.

\section{REFERENCES}

BCHM10 C. Birkar, P. Cascini, C. D. Hacon, and J. MKernan, Existence of minimal models for varieties of log general type, J. Amer. Math. Soc. 23 (2010), no. 2, 405-468; doi:10.1090/S0894-034709-00649-3.

Bir16 C. Birkar, Existence of flips and minimal models for 3-folds in char p, Ann. Sci. Éc. Norm. Supér. (4) 49 (2016), no. 1, 169-212; doi:10.24033/asens. 2279.

BW17 C. Birkar and J. Waldron, Existence of Mori fibre spaces for 3-folds in char $p$, Adv. Math. 313 (2017), 62-101; doi:10.1016/j.aim.2017.03.032.

CGS16 P. Cascini, Y. Gongyo, and K. Schwede, Uniform bounds for strongly F-regular surfaces, Trans. Amer. Math. Soc. 368 (2016), no. 8, 5547-5563; doi:10.1090/tran/6515.

CP08 V. Cossart and O. Piltant, Resolution of singularities of threefolds in positive characteristic. I. Reduction to local uniformization on Artin-Schreier and purely inseparable coverings, J. Algebra 320 (2008), no. 3, 1051-1082; doi:10.1016/j.jalgebra.2008.03.032.

CP09 - Resolution of singularities of threefolds in positive characteristic. II, J. Algebra 321 (2009), no. 7, 1836-1976; doi:10.1016/j.jalgebra.2008.11.030. 


\section{HACON AND J. WitASZEK}

CR15 A. Chatzistamatiou and K. Rülling, Vanishing of the higher direct images of the structure sheaf, Compos. Math. 151 (2015), no. 11, 2131-2144; doi:10.1112/S0010437X15007435.

CvS09 S. Cynk and D. van Straten, Small resolutions and non-liftable Calabi-Yau threefolds, Manuscripta Math. 130 (2009), no. 2, 233-249; doi:10.1007/s00229-009-0293-0.

CT16 P. Cascini and H. Tanaka, Purely log terminal threefolds with non-normal centres in characteristic two, Amer. J. Math., to appear, arXiv:1607.08590.

CT18_ Smooth rational surfaces violating Kawamata-Viehweg vanishing, Eur. J. Math. 4 (2018), no. 1, 162-176; doi:10.1007/s40879-016-0127-z.

CTW17 P. Cascini, H. Tanaka, and J. Witaszek, On log del Pezzo surfaces in large characteristic, Compos. Math. 153 (2017), no. 4, 820-850; doi:10.1112/S0010437X16008265.

CTW18_ Klt del Pezzo surfaces which are not globally F-split, Int. Math. Res. Not. 2018 (2018), no. 7, 2135-2155; doi:10.1093/imrn/rnw300.

CTX15 P. Cascini, H. Tanaka, and C. Xu, On base point freeness in positive characteristic, Ann. Sci. Éc. Norm. Supér. (4) 48 (2015), no. 5, 1239-1272; doi:10.24033/asens. 2269.

Cut09 S. D. Cutkosky, Resolution of singularities for 3-folds in positive characteristic, Amer. J. Math. 131 (2009), no. 1, 59-127; doi:10.1353/ajm.0.0036.

Das15 O. Das, On strongly F-regular inversion of adjunction, J. Algebra 434 (2015), 207-226; doi: 10.1016/j.jalgebra.2015.03.025.

ES80 G. Ellingsrud and T. Skjelbred, Profondeur d'anneaux d'invariants en caractéristique $p$, Compos. Math. 41 (1980), no. 2, 233-244; http://www.numdam.org/item?id=CM_1980__41_2_ 233_0.

GNT15 Y. Gongyo, Y. Nakamura, and H. Tanaka, Rational points on log fano threefolds over a finite field, J. Eur. Math. Soc., to appear, arXiv:1512.05003.

Har98 N. Hara, Classification of two-dimensional F-regular and F-pure singularities, Adv. Math. 133 (1998), no. 1, 33-53; doi:10.1006/aima.1997.1682.

HX15 C.D. Hacon and C. Xu, On the three dimensional minimal model program in positive characteristic, J. Amer. Math. Soc. 28 (2015), no. 3, 711-744; doi:10.1090/S0894-0347-201400809-2.

KM98 J. Kollár and S. Mori, Birational geometry of algebraic varieties, Cambridge Tracts in Math., vol. 134 (Cambridge Univ. Press, Cambridge, 1998); doi:10.1017/CB09780511662560.

Kol92 J. Kollár (ed.), Flips and abundance for algebraic threefolds (Soc. Math. de France, Paris, 1992); doi:10.1017/CB09780511662560.

Kol13 _ Singularities of the minimal model program, Cambridge Tracts in Math., vol. 200 (Cambridge Univ. Press, Cambridge, 2013); doi:10.1017/CB09781139547895.

Kov17 S. J. Kovács, Super-rational singularities, 2017, arXiv:1703.02269v3.

Kov18 , Non-Cohen-Macaulay canonical singularities, in Local and Global Methods in Algebraic Geometry, Contemp. Math., vol. 712 (Amer. Math. Soc., Providence, RI, 2018), 251-259; doi: 10.1090/conm/712/14349.

Lip78 J. Lipman, Desingularization of two-dimensional schemes, Ann. Math. 107 (1978), no. 1, 151207; doi:10.2307/1971141.

LS14 C. Liedtke and M. Satriano, On the birational nature of lifting, Adv. Math. 254 (2014), 118137; doi:10.1016/j.aim.2013.10.030.

PS14 Z. Patakfalvi and K. Schwede, Depth of F-singularities and base change of relative canonical sheaves, J. Inst. Math. Jussieu 13 (2014), no. 1, 43-63; doi:10.1017/S1474748013000066.

SS10 K. Schwede and K. E. Smith, Globally F-regular and log Fano varieties, Adv. Math. 224 (2010), no. 3, 863-894; doi:10.1016/j.aim.2009.12.020.

ST12 K. Schwede and K. Tucker, A survey of test ideals, in Progress in Commutative Algebra 2 (Walter de Gruyter, Berlin, 2012), 39-99. 


\section{ON THE RATIONALITY OF KLT SINGULARITIES}

Tan14 H. Tanaka, Minimal models and abundance for positive characteristic log surfaces, Nagoya Math. J. 216 (2014), 1-70; doi:10.1215/00277630-2801646.

Tan18_, Minimal model program for excellent surfaces, Ann. Inst. Fourier (Grenoble) 68 (2018), no. 1, 345-376; doi:10.5802/aif.3163.

Xu14 C. Xu, Finiteness of algebraic fundamental groups, Compos. Math. 150 (2014), no. 3, 409-414; doi:10.1112/S0010437X13007562.

Yas14 T. Yasuda, The p-cyclic McKay correspondence via motivic integration, Compos. Math. 150 (2014), no. 7, 1125-1168; doi:10.1112/S0010437X13007781.

Christopher Hacon hacon@math.utah.edu

Department of Mathematics, University of Utah, Salt Lake City, UT 84112, USA

Jakub Witaszek jakubw@umich.edu

Department of Mathematics, Imperial College, London, 180 Queen's Gate, London SW7 2AZ, UK

Current address: Department of Mathematics, University of Michigan, 2074 East Hall, 530 Church Street, Ann Arbor, MI 48109-1043, USA 